\title{
INTERNALISMO E EXTERNALISMO EM LINGUÍSTICA E A NEUROCIÊNCIA DA LINGUAGEM
}

\section{Marcello MODESTO*}

- RESUMO: O objetivo principal deste trabalho é discutir a possibilidade de investigação da realidade neurofisiológica da sintaxe das línguas naturais. Para que tal estudo seja possível, linguistas e neurocientistas teriam que achar uma base epistemológica comum. Entretanto, a visão prevalente em neurociência, que assume aprendizado associativo por correlação, não se combina bem com a visão internalista da sintaxe, tomada por linguistas gerativos. Em virtude disso, este trabalho discute as bases epistemológicas das teorias linguísticas mais aceitas atualmente, servindo de guia para neurocientistas que queiram investigar o processamento da linguagem pelo cérebro. Por outro lado, o artigo discute pesquisas desenvolvidas pela neurociência da linguagem para que os linguistas possam avaliar os problemas que surgem na intersecção das duas áreas. Conclui-se que qualquer pesquisador deve ter um entendimento claro dessas questões epistemológicas antes de montar qualquer experimento sobre a linguagem; e que qualquer conclusão acerca da realidade neural da sintaxe é, no presente, difícil de ser mantida.

- PALAVRAS-CHAVE: Linguística. Cognição. Neurociência. Sintaxe.

\section{Preâmbulo: objetivos}

Este trabalho tem dois objetivos principais. Por um lado, o artigo discute as bases epistemológicas das teorias mais representativas da linguística atual, servindo assim como um guia para pesquisadores de outras áreas interessados em responder como a língua é processada pelo cérebro; como é adquirida por falantes; em que medida o ambiente externo determina a competência final de um falante adulto; e outras questões. Por outro lado, discute alguns trabalhos recentes da neurociência da linguagem, servindo assim como um guia para linguistas interessados em investigar a realidade neurofisiológica da sintaxe. Dessa maneira, este estudo espera contribuir para uma possível colaboração entre linguistas e neurocientistas na elaboração de experimentos.

A seção 2 introduz os fatos mentais como objeto de estudo científico e a relação entre as diversas ciências que se ocupam desses fatos. Introduz também

* USP - Universidade de São Paulo. Faculdade de Filosofia, Letras e Ciências Humanas. São Paulo - SP - Brasil. 05508-900-modesto@usp.br 
a tensão entre a perspectiva nativista e a perspectiva empiricista, que incide sobre os estudos da cognição humana e que será um tema recorrente neste trabalho. A seção 3 procura descrever o "fenômeno linguístico", a dificuldade de sua delimitação e a tensão entre uma perspectiva internalista e uma externalista quanto ao objeto de estudo da linguística, outro tema recorrente neste trabalho. A seção 4 retoma o problema da delimitação do objeto de estudo da linguística e discute sua formulação em Saussure e Chomsky, com o intuito de discutir as bases epistemológicas das teorias atuais que se embasam no trabalho desses grandes pesquisadores. A seção 5 discute alguns trabalhos desenvolvidos pela neurociência da linguagem e a sua interpretação quanto às tensões nativismo vs. empiricismo e internalismo vs. externalismo. A seção 6 traz à tona uma teoria linguística que parece se adequar melhor à visão prevalente na neurociência, a linguística cognitiva, e discute alguns problemas dessa teoria. Finalmente, a seção 7 conclui o trabalho.

\section{Introdução: o estudo da mente}

O estudo da linguagem humana está intrinsecamente ligado ao estudo da mente e ao estudo da cognição, que é parte do primeiro, apesar disso não parecer óbvio a todo linguista. A princípio, a mente é estudada pela psicologia, mas a antropologia, quando estuda as sociedades, os ritos e os costumes, também estuda fatos essencialmente mentais. Lévi-Strauss (1949), por exemplo, entende a cultura de um grupo como um sistema compartilhado e estruturado por princípios, o que se assemelha bastante com a visão de sistema linguístico proposta por Saussure (1971) em seu Curso de linguística geral, como se verá adiante. De fato, as relações entre as teorias de Lévi-Strauss e as de Saussure e a linguística estruturalista são bem conhecidas. As mesmas tensões estão presentes no trabalho de ambos: tanto a língua como a cultura podem ser tomadas como objetos internos ou externos aos indivíduos. Se tomada numa perspectiva internalista, a existência de princípios universais que organizam as sociedades e as línguas levanta a questão sobre a existência de princípios gerais que organizam a mente humana. A psicologia provavelmente diria que há realmente princípios universais que organizam o pensamento e as emoções humanas (ambos fatos mentais). Os princípios que organizam o pensamento, entretanto, foram mais estudados até hoje pela filosofia (pela lógica e pela filosofia da mente). $O$ fato de que a linguística teria muito a contribuir na revelação desses princípios que norteiam o pensamento e a cognição humana leva Chomsky (2000a) e outros (CHOMSKY, 2011; CHOMSKY; BERWICK, no prelo), por exemplo, a encararem as línguas como objetos de estudo da psicologia e, em última análise, da biologia. 
O estudo da cognição é uma parte crucial do estudo da mente, que não é um objeto estático e se transforma de acordo com a experiência. Novamente, a psicologia abraça esses objetos de estudo. Numa perspectiva metateórica, a psicologia deveria também explicar o estado inicial da mente. ${ }^{1}$ No contexto deste trabalho, a questão mais crucial é se o ser humano nasce uma tabula rasa ou já vem equipado por instintos e predisposições. Se a segunda possibilidade for verdadeira, o que parece consensual, há de se descrever como essas predisposições interagem com a experiência para produzir um produto final (a mente de um indivíduo adulto). Seria interessante saber, principalmente, em que medida interagem. Quanto do conhecimento de Pedro advém dessas predisposições inatas e quanto da interação com o mundo exterior, na aprendizagem. O exemplo da aprendizagem tem de ser tomado aqui de uma maneira ampla: desde que nascemos, aprendemos uma língua, matemática, costumes, crenças, e inúmeras outras coisas. Quanto a crenças, pode-se objetar que não as aprendemos, nós as herdamos ou as absorvemos (ou as criamos novas). Para a linguagem, podese objetar o mesmo: a língua é de alguma forma absorvida (dada pelo ambiente social) e não aprendida. Isso torna ainda mais importante que, para cada um desses "aprendizados", explique-se como acontecem: quanto do aprendizado se deve a recursos geneticamente fornecidos a nós e quanto se deve a fatores externos.

O fato de a língua ser absorvida (ou adquirida, como se diz comumente em linguística) ou "depositada no cérebro" do indivíduo, como disse Saussure, torna a possibilidade de uma predisposição interna ainda mais plausível. Mesmo o aprendizado de matemática, que poderia parecer a priori um produto exclusivo da experiência do indivíduo, pode ser considerado como envolvendo faculdades naturais do ser humano. Chomsky (em PIATTELLI-PALMARINI, 1980, p.321) considera " $[. .$.$] a habilidade humana de lidar com propriedades profundas do$ sistema numérico." e supõe que essa faculdade seja "geneticamente determinada para os humanos." Na verdade, autores como Chomsky e Fodor (1975), entre outros, tomam como fato autoevidente que não se pode aprender nada, a menos que alguns "preconceitos" inatos estejam disponíveis. Hilary Putnam (apud PIATTELLI-PALMARINI, 1980, p.301) concorda que "[...] é verdade que nós não podemos aprender como aprender a menos que nós tenhamos alguma predisposição para aprender: nós precisamos ter alguma predisposição para aprender que não seja ela mesma aprendida, ou teríamos um regresso infinito."

Entre Putnam e Chomsky, o debate gira em torno de explicar o conhecimento atingido por um indivíduo normal munido apenas de "inteligência", ou munido de vários mecanismos especializados. Putnam argumenta contra esses mecanismos

Freud (1996) tenta explicar o estado inicial da mente, fazendo uma analogia com o conhecimento instintivo dos animais. Ele diz que, na nossa mente, há um fator instintivo, que seria o núcleo do inconsciente, um tipo primitivo de atividade mental, que seria encoberto e destorcido pela razão humana, quando essa faculdade é adquirida pelo homem. 
especializados e assume um mínimo de naturismo (que parece inescapável, como discutido acima): "So far I have assumed that there is such a thing as general intelligence; that is, that whatever else our innate cognitive repertoire may include, it must include multipurpose learning strategies, heuristics, and so forth" (PUTMAN, 2011, p.403). A visão oposta é advogada pelo neurocientista cognitivista C. R. Gallistel, mencionado por Chomsky (2002, p.84-85) nesta passagem:

[Gallistel holds] the substantive theory that in all animals, learning is based on specialized mechanisms, "instincts to learn" in specific ways; what Tinbergen called "innate dispositions to learn." These "learning mechanisms" can be regarded as "organs within the brain [that] are neural circuits whose structure enables them to perform one particular kind of computation," as they do more or less reflexively apart from "extremely hostile environments." Human language acquisition is instinctive in this sense, based on a specialized "language organ." [...] "To imagine that there exists a general purpose learning mechanism in addition to all these problem-specific learning mechanisms [...] is like trying to imagine the structure of a general purpose organ, the organ that takes care of problems not taken care of by adaptively specialized organs like the liver, the kidney, the heart and the lungs," or a "general purpose sensory organ, which solves the problem of sensing" for the cases not handled by the eye, the ear, and other specialized sensory organs. Nothing like that is known in biology.

Se, por um lado, a linguística relaciona-se ao estudo da mente, com todas as suas subdivisões; a mente e, por conseguinte, a linguagem, também teriam de ser explicadas numa dimensão fisiológica e biológica. Nesse sentido, queremos saber: como a língua (e outros fatos mentais) se instancia no cérebro por meio da transmissão de pulsos elétricos entre células nervosas; como e por que a evolução da nossa espécie culminou apresentando os fatos desta maneira e não daquela. Sobre a primeira questão, Chomsky (2002) é pouco otimista e sua posição contrasta com duas visões muito difundidas em neurociência. A posição chomskyana, que é de alguma forma ratificada por este trabalho, é a de que o nosso "[...] entendimento atual é bem insuficiente para deitar as bases de uma unificação entre as ciências do cérebro e as faculdades mentais mais elevadas, a linguagem sendo uma delas." (CHOMSKY, 2002, p.61, tradução nossa). As posições contrastantes em neurociências são: o modelo computacional da mente (BLOCK, 1990; JOHNSON-LAIRD, 1988) e as correntes que tomam a linguagem como um objeto extra-humano (presentes, segundo Chomsky, não só na neurociência, como também na filosofia da mente e na etologia) (DEACON, 1998). Chomsky salienta que a ideia (atribuída ao neurocientista Vernon Mountcastle) de que "[... as

No original: "[...] current understanding falls well short of laying the basis for the unification of the sciences of the brain and higher mental faculties, language among them." 
coisas mentais e, de fato, as mentes, são propriedades emergentes dos cérebros."3 (CHOMSKY, 2002, p.55, tradução nossa), mais do que apresentar uma solução para o problema, simplesmente reitera o que já era sabido pelos filósofos do século XVIII, para quem as propriedades "chamadas mentais" são "[...] o resultado de uma estrutura orgânica" do cérebro e "do sistema nervoso humano" em geral (CHOMSKY, 2002, p.70, tradução nossa).

Sobre a segunda questão, há uma vasta literatura sobre a gênese da linguagem incluindo algumas visões explícitas de Chomsky (HAUSER; CHOMSKY; FITCH, 2002), o qual tem uma visão saltacional, em que, após modificações auxiliares (e independentes da linguagem) terem acontecido na linhagem humana, a linguagem (em verdade, a sintaxe) surge de uma modificação genética pequena, mas com grandes consequências. Derek Bickerton $(1990,1995)$ descreve um possível cenário em que, numa primeira etapa, o léxico humano é criado e aumentado impulsionado pelo uso de uma protolíngua pelos homens primitivos, e esse léxico fornece um campo fértil para o nascimento, num segundo momento, da sintaxe (que resulta nas línguas modernas).

Além de lidar com esses fatos essencialmente linguísticos, a teoria da mente ainda tem que lidar com outros dois objetos que não se ligam tão diretamente à linguagem. Um é conhecido como o "difícil problema da consciência" (CHURCHLAND, 1984; SHEAR, 1997; CHALMERS, 1995; DENNETT, 1991, 2003); o outro constitui a atenção. Uma das metáforas mais sedutoras e produtivas em neurociência é tomar o cérebro como um computador, que executa subrotinas "aprendidas" através da experiência (talvez por meio de mecanismos inatos especializados). Para alguns pesquisadores, não seria exagero comparar a língua com uma dessas sub-rotinas executadas pelo cérebro. O que parece difícil de explicar é o fato de esse computador ser consciente de si mesmo. Um computador, quando executa sub-rotinas, não tem consciência de estar fazendo isso, nem pode refletir sobre aquilo que executa. Apesar de toda reflexão envolver linguagem, a consciência de estar agora sentado não é propriamente linguística, até o momento em que eu penso (desloco minha atenção) sobre estar sentado. Consciência e atenção parecem ser as faculdades mais elevadas, acima até mesmo da linguagem. São elas que me permitem pensar sobre a palestra que estou indo dar, enquanto consciente de estar dirigindo meu carro, da distância dos outros carros do meu, do semáforo que está verde, e de uma série de outros fatos; assim como também o que me permite abandonar esses pensamentos quando uma bola atravessa o caminho em frente ao carro. De repente, o ato de dirigir, que cozinhava numa consciência em segundo plano (sem atenção), pula para uma consciência plena (com atenção), que me permite frear rapidamente na expectativa de que alguma criança esteja correndo atrás da bola (BICKERTON, 1995; JACKENDOFF,

No original: "Things mental, indeed minds, are emergente properties of brains." 
2002). Obviamente, não temos a menor ideia de como esses fenômenos acontecem na dimensão neurológica, apesar de vivenciarmos esses fatos na esfera mental cotidianamente. Derek Bickerton (1995) defende que é a linguagem que possibilita aos humanos usufruir desse tipo de consciência reflexiva que falta às máquinas e aos animais. As seções que se seguem buscam, portanto, localizar a linguística (e seu objeto de estudo) em meio a todos esses fatos mentais.

A discussão que se segue jamais poderia fazer jus, ou sequer mencionar, todos os aspectos e questões relacionadas a todos esses assuntos. Iremos discutir mais propriamente o equilíbrio entre pressupostos nativistas e empiricistas em três grandes correntes linguísticas: uma que parte de Saussure e é conhecida como estruturalismo; uma que parte de Chomsky e é conhecida como gerativismo; e outra que parte de gerativistas dissidentes (George Lakoff e Charles Fillmore, entre outros) e é conhecida como cognitivismo. O estruturalismo toma a língua e os discursos produzidos com ela como objetos externos aos indivíduos e, portanto, não tomam partido sobre o quanto é inato e o quanto é adquirido (a questão não é pertinente e nem mesmo surge nos trabalhos dessa linha teórica). O gerativismo, grosso modo, parte do pressuposto de que há um componente inato especializado que auxilia na aquisição de uma ou mais línguas. O cognitivismo, por sua vez, toma como inato apenas estratégias gerais de aprendizagem e computação cujo propósito é múltiplo, ou seja, não especializadas para a linguagem. Deve ser ressaltado que essa teoria propõe que esses princípios gerais de computação podem ser verificados por fatos linguísticos, mas atestam sobre o funcionamento do pensamento. A hipótese não é trivial, como será discutido adiante.

A seção seguinte discute o estruturalismo descendente de Saussure e os fatos da linguagem. Notamos, novamente, que, enquanto discutimos Saussure, queremos discutir as bases epistemológicas tomadas pelas teorias que se fundam na ideia de língua como sistema, fato social, e não propriamente o trabalho daquele autor em particular. A semântica estrutural e a semiótica greimasiana, que decorre daquela, por exemplo, é uma teoria da significação que deixou de lado a relação entre a linguagem e o pensamento em virtude de tomar a língua como produto histórico-social. A discussão que segue mostra que essa tomada de posição não pode ser atribuída a Saussure e que a relação citada não está (ou não deveria estar em princípio) fora do alcance do estruturalismo.

\section{O fenômeno linguístico}

Saussure se referia ao fenômeno linguístico como o "conjunto heteróclito dos fatos da linguagem". A importância (e a extensão) desse fenômeno fica destacada quando se utiliza um exercício de imaginação muito usado por Chomsky (CHOMSKY, 2000a, 2000b; HAUSER; CHOMSKY; FITCH, 2002; PIATTELLIPALMARINI, 1980; entre outros): 
If a Martian graced our planet, it would be struck by one remarkable similarity among Earth's living creatures and a key difference. Concerning similarity, it would note that all living things are designed on the basis of highly conserved developmental systems that read an (almost) universal language encoded in DNA base pairs. [...] If our Martian naturalist were meticulous, it might note that the faculty mediating human communication appears remarkably different from that of other living creatures. (HAUSER; CHOMSKY; FITCH, 2002, p.1569).

Um cientista marciano meticuloso poderia concluir que, diferentemente de outros animais, que podem comunicar diversas coisas de fato, o ser humano possui a capacidade de transmitir seus pensamentos a outros indivíduos da espécie. Nenhum outro animal do planeta sequer se aproxima de tal façanha: ser capaz de traduzir seu pensamento em uma linguagem que possa ser transmitida por ondas sonoras (ou por gestos). Os animais não possuem linguagem (no sentido usado aqui), exatamente porque ela não é um sistema de comunicação (que os animais possuem, dos mais variados tipos), mas um sistema de expressão do pensamento. Linguagem e pensamento estão intimamente ligados. Apesar de nos parecer correto dizer que um animal relativamente complexo (meu cão, por exemplo) pensa, mesmo que de uma forma rudimentar, há uma diferença qualitativa entre o pensamento dos humanos e o dos outros animais (o que Bickerton (1995) chama de consciência 1 e consciência 2).

A capacidade, por exemplo, de considerar, discutir e informar outros indivíduos sobre eventos que acontecem em espaços ou pontos no tempo remotos é uma capacidade unicamente humana. O único sistema de comunicação do reino animal que parece ter algo remotamente similar a essa propriedade de deslocamento (no espaço, embora não no tempo) é a dança usada pelas abelhas para indicar às outras abelhas da colmeia a localização, o tipo e a quantidade de alimentos. É importante notar que não há deslocamento temporal (uma abelha nunca dança sobre a comida de ontem) e que a amplitude de representação do mundo é restrita (a dança de uma abelha é sempre sobre comida, sua localização, tipo e quantidade), enquanto a linguagem é irrestrita em ambos os sentidos. O termo linguagem, portanto, destina-se exclusivamente aos sistemas de representação humanos. Num sentido amplo, o termo abarca sistemas de comunicação: a música, o código morse, etc.); num sentido mais estrito, a linguagem (o mais apropriado seria dizer a faculdade da linguagem) abarca os sistemas linguísticos (verbais ou de sinais), que são sistemas de representação do pensamento, como discutido.

Quando Saussure se refere ao "conjunto heteróclito dos fatos da linguagem", o que ele tem em mente é exatamente o processo pelo qual o pensamento de um indivíduo A é transmitido a um indivíduo B por meio de ondas sonoras. ${ }^{4}$ Esse

4 Como deixa claro o diagrama no terceiro capítulo do Curso de linguística geral (SAUSSURE, 1971, p.19). 
processo envolve, necessariamente, uma espécie de tradução do pensamento em estruturas linguísticas; a tradução dessas estruturas em comandos motores (que movimentam os músculos); a recepção do som pelo outro indivíduo e uma tradução reversa para chegar finalmente ao significado daquele som produzido por A. Para estudar esse fenômeno notável, teríamos de apelar a um grupo (relativamente grande) de cientistas marcianos: psicólogos, neurocientistas, biólogos, linguistas, antropólogos. Entretanto, Saussure parece incluir no conjunto de fatos da linguagem ainda mais elementos. Quando se indaga sobre uma ciência geral dos sistemas de signos, a semiologia, Saussure inclui também como objeto de interesse científico os ritos e os costumes sociais.

Saussure escolhe estudar uma parte desse fenômeno complexo de tradução do pensamento: a língua (o código social de signos que possibilita todo o processo), em virtude de ser um objeto de estudo homogêneo e estável. Entretanto, não se deve concluir que o pai da linguística desprezava os outros componentes do fenômeno linguístico. Isso se verá com mais detalhes na seção seguinte.

A fábula do marciano coloca o problema numa perspectiva didática. 0 marciano pode recortar teoricamente o sistema linguístico e os textos produzidos por esses animais, os humanos, e estudar esses objetos separadamente; mas o marciano poderia também se perguntar sobre como o ser humano pensa e como esse pensar se relaciona com o comportamento (o agir) e com a própria linguagem. O marciano notaria que a propriedade principal desses "textos" (que podem consistir de apenas uma frase ou expressão) é conter um significado. O estudo da significação, portanto, está na base de qualquer estudo sobre a linguagem, a mente ou o pensamento, e linguistas e filósofos já escreveram muito sobre isso. O estudo da significação abre as portas para outras questões fundamentais: como o ser humano constrói um sistema de crenças sobre o mundo exterior e o mundo interior? Como o sistema linguístico influi, modifica, constrói esse sistema de crenças, ou, dito de outro modo, o sistema linguístico influencia de alguma forma na visão de mundo do indivíduo? Até hoje, a linguística apenas arranhou a superfície dessas questões.

Uma tese promissora nesse contexto manteria que, se o homem produz significação a partir da realidade exterior, estudando a realidade exterior e como ela é interpretada pelo homem, estaríamos também estudando um processo mental de construção da realidade (uma realidade significante). A base dessa tese é o fato de que a realidade existe para o ser humano na medida em que ele pode reconstituí-la por meio dos sentidos e interpretar um significado nesse percepto construído. A estruturação da realidade para o homem é essencialmente linguística. O homem olha para o mundo e vê mesas, cachimbos e árvores, quando na verdade o que existem são objetos que emanam luz, que têm uma certa proporção e um certo formato. Num sentido, não existem 
cachimbos (nem árvores) no mundo real. O que existem são objetos construídos pelo homem, que são interpretados como cachimbos e objetos naturais dos mais variados e que são agrupados pelo homem em classes como árvore, arbusto, etc. Alguém que nunca viu um cachimbo e não tem na sua língua uma palavra para cachimbo veria apenas um objeto, e poderia atribuir a esse objeto usos inusitados (como bater na cabeça das pessoas ou levar comida à boca). O mundo exterior é o mesmo, mas as interpretações sobre esse mundo variam de indivíduo para indivíduo e isso se deve em grande parte à língua. Esse é um caráter cultural da língua.

A linguística, tradicionalmente, estuda o sistema que estrutura a significação produzida pelo homem, deixando a interpretação do mundo real para outras áreas. Entretanto, a construção do mundo mental (que inclui uma representação do mundo externo) é essencialmente linguística: a partir de um certo cenário (e motivado por uma comunidade humana) Pedro constrói o significado de cachimbo. Cachimbo passa então a fazer parte da realidade de Pedro. Nesse sentido, os objetos são o resultado das instanciações ${ }^{5}$ feitas pelos humanos dos conceitos e, de certa forma, representam os conceitos. Do mesmo modo que um som, que compõe um enunciado, é, de certa maneira, uma representação, ou uma instância de uma unidade abstrata que é o fonema, os objetos instanciam e representam os conceitos. O som pode variar bastante (incluindo, às vezes, sons completamente diferentes que representam a mesma unidade fonológica), mas a interpretação continua constante. O mesmo se dá com árvores e cachimbos, ou seja, forma, cor e uso dos objetos podem variar bastante, mas o significado continua constante.

O que se pode concluir por hora é que o objeto de estudo saussureano é um recorte teórico que possibilita o estudo de um processo tão extenso que pode ser estudado ao mesmo tempo pela psicologia, fisiologia, antropologia, biologia, sociologia, etc., como se verá com mais detalhe na seção a seguir. O delineamento de um objeto de estudo, entretanto, não deve impedir ou sombrear o delineamento de outra parte desse grande processo da linguagem. Ou seja, as teorias que tomam o objeto de estudo saussureano e discutem, principalmente, a significação não deveriam ser tomadas como irrelevantes com relação ao estudo da mente. Assim, se a significação é bem definida em alguma teoria linguística numa perspectiva externalista, isso deve ser usado para que se explique como a significação acontece numa perspectiva internalista, que ainda parece muito pouco definida. Retomamos essas questões na seção seguinte.

5 Apesar do uso do verbo instanciar não estar completamente estabelecido em português, não me ocorre outra maneira de diferenciar "instanciação" (o ato de instanciar, de criar uma instância de um objeto) de "instância" (o produto final produzido pela instanciação). 


\section{O objeto da linguística: de Saussure a Chomsky}

Em um episódio de um seriado cômico da TV britânica, um chef de cozinha está servindo bifes aos comensais, enquanto sua ajudante serve o acompanhamento. Num certo momento, a ajudante (marcada abaixo como G, de garota) pergunta ao chef (marcado C) o que a palavra steak significa. O diálogo está transcrito abaixo e esse trecho do programa pode ser visto no site YouTube:

G: Chef, what does steak mean?

C: Pardon?

G: What does steak mean?

C: (showing a piece of meat) This is steak.

G: Yes, but what does it actually mean, the word?

C: Well, it's a kind of meat, isn't it?

G: Not always.

C:Hum?

G: Well, you've got fish steaks, haven't you?

C: Okay, good point.

G: Salmon steaks.

C: As I say...

G: Wooden stakes.

C: Completely different type of steak, it's spelled...

G: High stakes.

C: Right, well, that's completely different...

G: Stake through the heart.

C: No, it's still...

G: Stake and Ale pie.

C: Well, okay now we're back to the first one, which is clearly meat.

G: But what does it mean?

$C$ : (hanging the piece of meat in front of the girl's face and yelling) It means THIS! THIS IS STEAK!

Apesar de a garota confundir duas palavras homófonas (steak e stake), o que em parte produz a graça do sketch, ela está certa em não se contentar com a explicação dada pelo chef. Obviamente, o significado da palavra steak não é aquele pedaço de carne em particular que o chef está mostrando. Quando o chef mostra o pedaço de carne para a garota, ele está exemplificando um uso ou uma referência da palavra steak, mas não explicando o que a palavra significa. Um steak é geralmente um pedaço de carne cortada perpendicularmente à fibra da carne (no caso de carne bovina) ou perpendicularmente à espinha (no caso de peixe). Nesse sentido, steak se oporia a fillet (no caso de carne de peixe). 
Entretanto, a carne também pode estar moída, mantendo o formato de um bife (no caso de Salisbury steak e hamburguer steak), ou não (como no steak tartar). A carne também pode estar enrolada e cozida (swiss steak) ou desfiada dentro de uma torta (steak and ale pie), o que mostra que steak não pode ser simplesmente definido em relação à direção do corte da carne ou um formato específico. O significado de steak, portanto, parece envolver não só a direção do corte mas também um formato prototípico, entretanto ambos critérios podem estar ausentes. Pode-se dizer que, prototipicamente, steak se refere à carne bovina (uma vez que outros usos devem ser marcados explicitamente, como no caso de fish steak), tem o formato de um bife, etc. Entretanto, há objetos no mundo que, apesar de não corresponderem ao steak prototípico, podem ser chamados de steak. ${ }^{6}$

A posição tomada pelo chef, ou seja, a de tomar a referência da palavra como seu significado é praticamente a mesma das teorias conhecidas como semânticas formais (MONTAGUE, 1974; HEIM; KRATZER, 1998). Nessas teorias, baseadas principalmente na distinção fregueana entre sentido e referência, o significado de steak equivale ao conjunto de objetos (reais ou imaginários) que podem ser caracterizados como (chamados de) steak. Essas são, portanto, teorias que tomam o significado numa perspectiva externalista: o significado das unidades linguísticas é dado pelos objetos (reais ou imaginários) designados pelo termo e, portanto, é externo à linguagem. Dizendo de outro modo, a significação está no mundo ou, pelo menos, na relação entre a linguagem e o mundo. A posição tomada pela garota, por sua vez, é internalista no sentido que ela procura definir um significado que existe internamente à linguagem, dentro de um sistema linguístico de significados relacionados. Esse significado interno à língua é geralmente chamado de conceito (desde, pelo menos, Saussure (1971)). O conceito de steak, por exemplo, terá de ser maleável o suficiente para abarcar o significado de beefsteak, fishsteak, steak tartar e steak and ale pie; e, considerado internamente à língua, será definido por suas relações sintagmáticas e paradigmáticas com outros conceitos. ${ }^{7}$

Entretanto, se Saussure era um internalista quando à semântica, era também um externalista com relação à língua, o objeto de estudo da linguística. O valor de um signo (que abarca seu significado) só pode ser dado internamente à língua pois depende da rede de relações internas ao sistema. Bife e steak, por exemplo, podem ter um significado aproximado, mas têm valores bem diferentes: eu não posso (em português) comer um bife de peixe, nem fazer uma torta de bife. A língua, por outro lado, é tomada por Saussure como algo externo ao indivíduo (falante), como pode ser comprovado nesta passagem:

\footnotetext{
6 Sobre a teoria dos protótipos em ciência cognitiva, confira os trabalhos de Eleanor Rosch e colaboradores.

7 Confira o conceito de valor em Saussure (1971). Não é claro que a noção de conceito possa ser entendida exatamente como a intensão de um conjunto extensional. Parece haver bem mais em jogo aqui.
} 
[A Língua] não se confunde com a linguagem; é somente uma parte determinada, essencial dela, indubitavelmente. É, ao mesmo tempo, um produto social da faculdade de linguagem e um conjunto de convenções necessárias, adotadas pelo corpo social para permitir o exercício dessa faculdade nos indivíduos. [...] é a parte social da linguagem, exterior ao indivíduo, que, por si só, não pode nem criála nem modificá-la; ela não existe senão em virtude duma espécie de contrato estabelecido entre os membros de uma comunidade. (SAUSSURE, 1971, p.17 e p.22).

É importante que se entenda porque Saussure assim define a língua e porque toma esse objeto em particular (dentre tantos outros) como o objeto de estudo da linguística. É sabido, através do seu Curso de linguística geral, que Saussure se preocupava com a relação entre linguagem e pensamento. Entretanto, no começo do século XX, era impossível pretender explicar, ou mesmo estudar cientificamente todo o conjunto (heteróclito, segundo Saussure) dos fatos da linguagem (que envolve fenômenos inteiramente psíquicos, outros inteiramente fisiológicos, outros físicos, etc.). Dada essa impossibilidade, Saussure (1971, p.21) define um objeto concreto e homogêneo: a língua, "[...] um tesouro depositado pela prática da fala em todos os indivíduos pertencentes à mesma comunidade [...]", o código socialmente determinado. Essa tomada de posição teórica tem mais a ver com a possibilidade do estudo científico do que com a crença de que os sistemas linguísticos têm uma existência puramente social e não individual. Em verdade, para Saussure, o sistema linguístico existia, de alguma forma, no cérebro dos falantes:

Trata-se de [...] um sistema gramatical que existe virtualmente em cada cérebro ou, mais precisamente, nos cérebros dum conjunto de indivíduos, pois a língua não está completa em nenhum, e só na massa ela existe de modo completo. (SAUSSURE, 1971, p.21, grifo nosso).

[...] Os signos linguísticos, embora sendo essencialmente psíquicos, não são abstrações; as associações, ratificadas pelo sentimento coletivo e cujo conjunto constitui a língua, são realidades que têm sua sede no cérebro. (SAUSSURE, 1971, p.23, grifo nosso).

Logo antes de dizer que a língua é a parte social da linguagem, Saussure (1971, p.22) estranhamente afirma que "[...] pode-se localizá-la na porção determinada do circuito em que uma imagem auditiva vem associar-se a um conceito." Ora, mas essa porção está localizada entre a audição e a fonação no cérebro/mente dos falantes, segundo a ilustração de Saussure na página 20. Fica claro, portanto, que Saussure bem sabia que existe uma realidade psíquica e individual da linguagem, o que ele de fato afirmou explicitamente: "A linguagem tem uma lado individual e um lado social, sendo impossível conceber um sem o outro." (SAUSSURE, 1971, p.16). 
O linguista parece referir-se à parte individual da linguagem de duas maneiras. Uma é a fala, "um ato individual de vontade" (SAUSSURE, 1971, p.17), que pode ser estudada pela psicologia; ${ }^{8}$ a outra Saussure chama de faculdade

\section{da linguagem:}

[a língua é] um produto social da faculdade de linguagem [...] poder-se-ia objetar que o exercício da linguagem repousa numa faculdade que nos é dada pela Natureza, ao passo que a língua constitui algo adquirido e convencional, que deveria subordinar-se ao instinto natural em vez de adiantar-se a ele. [...] não é a linguagem que é natural ao homem, mas a faculdade de constituir uma língua, vale dizer: um sistema de signos distintos correspondentes a ideias distintas. [...] existe uma faculdade mais geral, a que comanda os signos e que seria a faculdade linguística por excelência. (SAUSSURE, 1971, p.17-18).

E conclui:

[...] uma faculdade de associação e de coordenação que se manifesta desde que não se trate mais de signos isolados; é essa faculdade que desempenha o principal papel na organização da língua enquanto sistema. (SAUSSURE, 1971, p.21).

Existe, portanto, para Saussure, uma faculdade "natural" da qual a língua é o produto. Apesar disso, a metáfora do jogo de xadrez, usada por ele, parece indicar que o linguista suíço equipararia o conhecimento que um falante tem da sua língua com o conhecimento que um jogador tem das regras do xadrez. Ambos os sistemas, o linguístico e o do jogo, seriam aprendidos por meio do contato com outros indivíduos que soubessem as "regras". Essa interpretação advém, exatamente, da perspectiva externalista que Saussure tem da língua.

Tomemos agora Chomsky, que toma a faculdade da linguagem como seu objeto de estudo. Em verdade, a "[...] faculdade de associação e coordenação que se manifesta desde que não se trate mais de signos isolados.", mencionada por Saussure (1971, p.21), e que "desempenha um papel principal na organização da língua enquanto sistema" parece muitíssimo com a definição chomskyana de gramática. Apesar de tomarem objetos de estudo completamente diferentes, não parece que Chomsky e Saussure diferem muito em suas interpretações dos fatos. Isso parece ter passado despercebido a grande parte dos linguistas. ${ }^{9}$

8 "Há o ponto de vista do psicólogo, o qual estuda o mecanismo do signo no indivíduo; é o método mais fácil, mas não ultrapassa a execução individual, não atinge o signo, que é social por natureza." (SAUSSURE, 1971, p.25).

9 Talvez as diferenças entre Saussure e Chomsky sejam mais importantes que suas semelhanças. Apesar disso, nossa intenção em discutir esses conceitos fundamentais é mostrar que mesmo as teorias externalistas de significação podem fornecer modelos explicativos de como o significado é produzido mentalmente (e que essa já era uma preocupação de Saussure). 
Chomsky toma a faculdade da linguagem como seu objeto de estudo. Sua teoria é, portanto, internalista: a linguagem existe na mente/cérebro do indivíduo (ou pelo menos uma parte dela). Chomsky entende essa faculdade como sendo a capacidade de Pedro de gerar um número possivelmente infinito de sentenças gramaticais na sua língua. Essa capacidade seria inata aos humanos e ausente nos outros animais. Isso explicaria porque o bebê Pedro adquire a língua de seus pais em apenas quatro anos de vida, mas o gatinho da família não, por mais estímulo linguístico que lhe seja dado. Usando as ideias de Gallistel (comentadas na introdução), Chomsky (2002, p.64) define assim a faculdade da linguagem:

This language organ, or "faculty of language" as we may call it, is a common human possession, varying little across the species as far as we know, apart from very serious pathology. [...] the language organ is the faculty of language (FL); the theory of the initial state of FL, an expression of the genes, is universal grammar (UG); theories of states attained are particular grammars; the states themselves are internal languages, "languages" for short. The initial state is, of course, not manifested at birth, as in the case of other organs, say the visual system.

Na passagem abaixo, Chomsky define as línguas particulares como, essencialmente, a sintaxe (um procedimento recursivo), interna ao falante, que "gera uma infinidade de expressões". É importante ressaltar também que Chomsky advoga por uma semântica internalista (como já havia feito explicitamente em outras ocasiões):

[...] we can think of a particular language $L$ as a state of FL. $L$ is a recursive procedure that generates an infinity of expressions. Each expression can be regarded as a collection of information for other systems of the mind-brain. [...] Each expression, then, is an internal object consisting of two collections of information: phonetic and semantic. These collections are called "representations," phonetic and semantic representations, but there is no isomorphism holding between the representations and aspects of the environment. There is no pairing of internal symbol and thing represented, in any useful sense. (CHOMSKY, 2000a, p.86-87).

Talvez não fique claro, nas passagens citadas, que, como a língua (L) é um estado da faculdade da linguagem (FL), se L é um procedimento recursivo, FL também deve ser um procedimento recursivo. De fato, desde Hauser, Chomsky e Fitch (2002), pelo menos, Chomsky explicitamente define a FL stricto sensu como um procedimento recursivo. Em resumo, Chomsky enxerga a sintaxe como um algoritmo que, dada uma sequência qualquer de unidades, produz uma estrutura hierárquica subjacente a essa sequência por meio de uma operação recursiva de juntar dois elementos. Por exemplo, para uma sequência de três itens lexicais a, b e c, a faculdade da linguagem geraria a estrutura abaixo, sendo que os rótulos 
dos objetos formados pela junção de dois itens ( $\alpha$ e $\beta$, na Figura 1) dependeriam da categoria morfológica atribuída a cada item lexical:

Figura 1 - Estrutura sintática formada pela operação JUNTAR

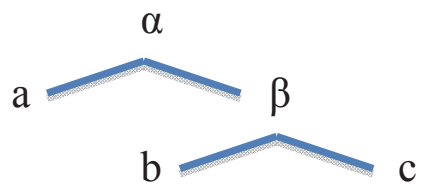

Fonte: Elaboração própria.

Uma possibilidade parece óbvia e talvez seja uma hipótese de trabalho frutífera: a faculdade da linguagem à qual Saussure se refere pode ser equacionada à sintaxe (o objeto de estudo chomskyano). Apesar de o humano somente adquirir uma língua quando em contato com outros humanos linguisticamente dotados, a teoria chomskyana prevê que, mesmo completamente sozinho, esse ser humano ainda teria esse algoritmo sintático, que lhe daria muitas vantagens no que diz respeito ao pensamento, ao planejamento de ações e à solução de problemas. Isso parece ser uma previsão acertada, afinal, um homem criado por lobos (sem língua) provavelmente não se tornaria menos humano.

Na perspectiva dessa teoria, então, uma língua não seria aprendida, apenas as correspondências entre significados e significantes são aprendidas e, por isso, o conhecimento que um falante tem de sua língua é distinto de qualquer outro conhecimento (como as regras do xadrez, por exemplo). Existe, portanto, na corrente gerativa, uma separação entre o léxico (que é aprendido) e a sintaxe (que é basicamente inata). Entretanto, a existência dessa separação entre léxico e sintaxe não é óbvia a todos os linguistas e, muito menos, aos neurocientistas.

Adotando-se a distinção entre léxico e sintaxe, ou não, nenhuma teoria linguística consegue, ainda, prover um modelo de como a mente/cérebro instancia o sistema linguístico individual. As considerações tecidas até aqui indicam que as teorias externalistas poderiam complementar a visão chomskyana, se tomadas numa perspectiva internalista. Os estudos da neurociência, discutidos a seguir, parecem confirmar essa hipótese.

\section{Neurociência}

Os experimentos feitos em neurociência que tomam as ideias chomskyanas como bases epistemológicas procuram elucidar como e onde esse procedimento recursivo se processa no cérebro (MORO, 2008; GRODZINSKY, 2006; BEN- 
SHACHAR; PALTI; GRODZINSKY, 2004; BEN-SHACHAR et al., 2003; MORO et al., 2001; INDEFREY et al., 2001; FRIEDERICI; PFEIFER; HAHNE, 1993; NEVILLE et al., 1991; entre outros). Ben-Shachar, Palti e Grodzinsky (2004), por exemplo, argumentam ter localizado uma área no cérebro que seria responsável pelo que chama de movimento sintático, ou "propriedade de deslocamento", que, segundo Chomsky, é característica das línguas. Apesar do otimismo de seus autores, é difícil acessar o que esses experimentos realmente mostram. Se algum experimento realmente registrasse um rastro de uma operação de ordem alta no cérebro (como fazer uma multiplicação, processar o significado de uma sentença ou planejar uma viagem), seria um avanço fantástico para a ciência. Estamos um pouco longe de poder fazer isso, como Chomsky (2000a, 2000b, 2002, entre outros) tem apontado insistentemente. O problema se deve, em grande parte, à diferente granularidade dos elementos conceituais usados pela linguística e pela neurociência, o que leva a uma incomensurabilidade ontológica (POEPPEL; EMBICK, 2005; EMBICK; POEPPEL, 2003).

Se o pesquisador toma outras bases epistemológicas que não aquelas da sintaxe gerativa, dificilmente procuraria encontrar a sintaxe "acontecendo" no cérebro. Para esses pesquisadores, talvez a maioria, o cérebro funciona por meio de correlações: "[...] padrões de atividade que coocorrem frequentemente [e] podem ser guardados de modo a reforçar os elos sinápticos entre os neurônios participantes." (PULVERMÜLLER, 2002, p.21-22, tradução nossa). Nesse sentido, o cheiro do café fica associado ao líquido e à situação de tomar café, uma vez que a atividade cerebral ligada ao cheiro coocorre frequentemente com atividades ligadas ao líquido e às situações. Essas correlações ficam, para usar um termo da linguística cognitiva, entrincheiradas no cérebro.

Segundo Pulvermüller (2002, p.62, tradução nossa), "[...] é amplamente reconhecido que o córtex motor é organizado somatotopicamente, isto é, músculos adjacentes estão representados em áreas cerebrais vizinhas do córtex motor."10 Do mesmo modo, existe uma divisão cortical entre modalidades (visual, olfativa, etc.) e, por isso, pode-se falar em córtex visual, motor, etc. As áreas do cérebro são interconectadas para permitir "um mapeamento complexo de padrões de informação entre as modalidades". Em resumo, podemos entender que "o córtex tem a função de juntar essas informações multimodais" (PULVERMÜLLER, 2002, p.22, tradução nossa) num todo coeso (o percepto aludido por Jackendoff (2002)). Aprendizagem por correlação também implica que "redes neurais distribuídas são formadas entre várias áreas corticais". Ainda nas palavras de Pulvermüller (2002, p.22):

10 Confira Penfield e Roberts (1959) e Penfield e Rassmussen (1950). 
Development of these networks, called functional webs, would be driven by sensorimotor or sensory-sensory coactivation, and would be determined by the available cortical projections indirectly connecting the coactivated neurons in primary areas to each other. [...] The cortex, a neuroanatomically defined associative memory obeying the correlation learning principle, allows for the formation of distributed functional webs. During language acquisition, the neurobiological principles governing the cortex interact to yield the neuron machinery underlying language.

Exemplificando, se o estímulo experimental for um garfo, espera-se que a parte do córtex motor, relacionada à boca, e o córtex ligado ao paladar tenham mais atividade do que o córtex visual, por exemplo (mesmo quando o estímulo apresentado é visual) (KIEFER; SPITZER, 2001; PULVERMÜLLER, 2001). O que é significativo para os linguistas é que, quando o estímulo é linguístico (quando o paciente ouve ou lê a palavra "garfo"), as mesmas regiões cerebrais são estimuladas. Parece haver muito pouca distinção cerebral entre ver um garfo, pensar num garfo, ler ou ouvir a palavra garfo. A distinção talvez seja apenas de grau, como demonstra De Araujo et al. (2012). Isso parece sugerir que há uma realidade neural para aquilo que Saussure chamava de "conceito". Obviamente, o conceito inclui (ou está indissociavelmente ligado a) uma imagem acústica. Ao vermos um garfo, a imagem acústica desse conceito sofre um priming, assim como outros conceitos que se relacionam paradigmaticamente com o primeiro. Segundo Pulvermüller (2002, p.24):

The cat concept would be realized as a large set of neurons distributed over a small set of cortical areas. All of these areas would serve as binding sites. A functional web will be assumed to be a set of neurons

(i) That are strongly connected to each other

(ii) That are distributed over a specific set of cortical areas

(iii) That work together as a functional unit

(iv) Whose major parts are functionally dependent on each other so that each of them is necessary for the optimal functioning of the web.

Estudos em neurociência têm confirmado que unidades linguísticas ativam as áreas neurais correspondentes ao seu significado. Assim, verbos do tipo "andar" evocam correntes mais fortes em áreas dorsais, vizinhas das áreas corticais correspondentes às pernas, enquanto verbos do tipo "falar" evocam correntes mais fortes em áreas inferiores, próximas às áreas que representam a face e os articuladores (lábios, língua) (HAUK; PULVERMÜLLER, 2004; PULVERMÜLLER; HUMMEL; HÄRLE, 2001; PULVERMÜLLER; HÄRLE; HUMMEL, 2000).

De acordo com Pulvermüller (2002, p.62), esses resultados " [...] dão suporte à tese de que a informação sobre a forma da palavra e as partes do corpo usadas para executar as ações relacionadas àquela palavra estão entrelaçadas na mesma rede 
cortical relacionada à palavra e são ativadas quase simultaneamente."11 $\mathrm{O}$ autor então conclui que "[...] o uso de uma palavra no contexto de objetos e ações leva a uma associação entre os neurônios na área cortical responsável pela linguagem e outros neurônios em áreas que processam informação sobre o referente das palavras.", assim o fato de haver "[...] conexões fortes dentro da rede pode explicar a impressão de que a imagem é automaticamente trazida pela forma da palavra apresentada sozinha e que, vice-versa, a imagem quase que automaticamente chama o nome para a memória ativa." (PULVERMÜLLER, 2002, p.56, tradução nossa). O autor então chama essa " [...] coleção de neurônios ligando informação fonológica e informação sobre as ações e as percepções a que uma palavra se refere" de redes lexicais (no original, word webs) (PULVERMÜLLER, 2002, p.56, tradução nossa). A modalidade da sensação ou da ação por meio da qual uma palavra é conhecida também é aparentemente relevante. Assim, palavras como "tubarão" e "baleia" evocam associações no córtex visual mais fortemente do que palavras como "gato", que são mais distribuídas, por razões óbvias (FUSTER, 1999; PULVERMÜLLER; LUTZENBERGER; PREISSL, 1999).

Já foi proposto, também, que o significado de uma palavra pode ser definido pelo conjunto de palavras que coocorrem frequentemente com aquela palavra (LANDAUER; DUMAIS, 1997), o que é muito próximo do entendimento saussureano dos conceitos lexicais. Além disso, a produção e compreensão de substantivos e de verbos, assim como de nomes de animais e ferramentas, foram afetados diferentemente por doenças do cérebro (BAK et al., 2001; CAPPA et al., 1998; WARRINGTON; MCCARTHY, 1983; WARRINGTON; SHALLICE, 1984). Ativação cortical diferenciada para conceitos relacionados a ações ou objetos é confirmada, em parte, por estudos que usam técnicas de imagem como PET e fMRI (NOPPENEY; PRICE, 2002; MOORE; PRICE, 1999; MUMMERY et al., 1998; GRABOWSKI; DAMASIO; DAMASIO, 1998; DAMASIO et al., 1996; WARBUNTON et al., 1996). Entretanto, não há consenso (DEVLIN et al., 2002). Em alguns estudos, não houve diferença na resposta cerebral entre verbos de ação e substantivos que evocam associações fortes a ações (PULVERMÜLLER; MOHR; SCHLEICHERT, 1999), o que leva a crer que a categoria gramatical da palavra importa menos do que seu significado (no léxico cerebral). Quanto à distinção entre itens lexicais funcionais (preposições, flexões verbais, etc.) e itens lexicais com conteúdo concreto, parece haver evidências, por meio de estudos de imagens neurofisiológicas, de que o processamento cortical dessas duas classes é diferente (BROWN; HAGOORT; TER KEURS, 1999; NOBRE; MCCARTHY, 1994; PULVERMÜLLER et al., 1996; SHTYROV; PULVERMÜLLER, 2002).

Considerando que a visão de que o cérebro constitui essas redes lexicais por correlações associativas parece bem estabelecida, percebemos a dificuldade de

11 Ver também Pulvermüller (2001). 
construir uma base teórica que permita a busca empírica sobre a realidade neural da sintaxe entendida como uma faculdade natural do ser humano. Por um lado, felizmente, o sistema de signos saussureano, a parte "social" da linguagem, parece estar bem representado no cérebro. A faculdade da linguagem, o procedimento recursivo aludido por Chomsky, por outro lado, parece bem mais arredio aos olhos humanos. Para que, a partir do que se sabe sobre como o léxico é instanciado no cérebro, seja possível uma averiguação científica da sintaxe, será necessário que um entendimento teórico acerca das perspectivas internalistas e externalistas evolua no sentido de possibilitar experimentos que localizem, comprovem ou falsifiquem a existência de uma sintaxe operada pelos neurônios. Se a sintaxe pode ser aprendida por correlação, então é necessário que se faça um esboço de como isso pode acontecer. Apesar de a teoria gerativa não se combinar muito bem com aprendizagem por correlação, existe uma outra teoria linguística, nomeada na introdução, que assume exatamente que a sintaxe (no sentido de ser um procedimento recursivo mental especializado para a linguagem) não existe e que talvez se possa adquirir a sintaxe da mesma maneira, grosso modo, que se adquire o léxico. Essa teoria será tratada a seguir.

Antes de passar a esse assunto, entretanto, é importante notar que os trabalhos comentados acima se restringem ao estudo da organização mental de itens lexicais (o que equivale ao sistema de signos saussureanos) e àqueles que tentam estabelecer correlatos neuronais da sintaxe (comentados brevemente no início desta seção). Em nenhum momento nos referimos a operações mentais mais complexas como inferências, estabelecimento de implicaturas e cálculos mentais que poderiam ser reconhecidos como pensamento. Isso se deve ao fato de esses assuntos estarem, atualmente, muito além do que a neurociência pode estudar. Salvo engano nosso, esses temas só têm sido desenvolvidos pela filosofia da mente e envolvem questões muito complexas (FODOR, 2000), que fogem ao escopo deste trabalho. Tudo o que se pode concluir da discussão aqui apresentada, portanto, é parcial, no sentido de que nosso conhecimento atual sobre a mente é sobremaneira parcial.

\section{Linguística cognitiva}

A chamada linguística cognitiva (LC) foi criada, entre outros, por Mark Johnson, George Lakoff e Charles Fillmore, os dois últimos gerativistas dissidentes. Apesar da dissidência ter se dado nos anos 60, a LC começa a ser reconhecida como área de pesquisa a partir do fim da década de 80 do século passado. Mais do que uma teoria concisa, a LC é um aglomerado de teorias que compartilham princípios e pressupostos comuns. A LC nega a separação chomskyana entre um léxico (aprendido) e uma sintaxe (inata) bem divididos e aposta num contínuo 
de construções. Apesar da definição de construção variar de autor para autor, construções prototípicas seriam aquelas já descritas pela gramática tradicional (construções passivas, bitransitivas, existenciais, etc.).

Como, nessa teoria, as construções são aprendidas por meio da experiência, esquemas sintáticos muito comuns (como, por exemplo, [NP V NP] em português ou inglês, que é instanciado por sentenças como "gatos tomam leite") também são considerados construções. Assim como os itens lexicais, as construções são dotadas de um significado (mesmo que esquemático). Essas construções seriam guardadas em uma rede mental de relações baseadas em prototipicidade e derivações metafóricas, às vezes chamada de constructicon (como em lexicon), um repositório de construções que variam de itens lexicais a esquemas sintáticos (dependendo da teoria escolhida).

Para exemplificar, muito superficialmente, o tipo de raciocínio usado na LC, tomemos duas construções do inglês: a construção de movimento causado (1a) e a construção resultativa (1b).

(1) a. George pushed Mary off the sofa.

b. George hammered the metal flat.

A primeira construção, a de movimento causado, tem um significado esquemático que envolve um ator agente (George), um tema (Mary) e um objetivo, ou goal, (off the sofa) e a ideia de movimento, que é dada pela própria construção. A construção resultativa pode ser vista como uma derivação metafórica da construção de movimento causado se tomarmos a metáfora "STATES are LOCATIONS" como um dos princípios que norteia a cognição humana do mundo. De fato, nos referimos a estados como se fossem lugares: entrar em depressão, cair num sono profundo, estar em paz, sair da pasmaceira. Se o estado de lisura (flatness) é interpretado metaforicamente como um objetivo do movimento causado por um agente, a construção resultativa realmente não difere da primeira. Isso fica especialmente claro em usos do tipo "George drank himself into oblivion", em que o agente George causa o movimento de um tema (himself) para dentro de um espaço metafórico (oblivion).

Nessa teoria, como o aprendizado se dá exclusivamente por meio da experiência, construções muito usadas ficam mais entrincheiradas (do inglês entrenched) no cérebro, assim como outras sequências (motoras, por exemplo: levar o garfo do prato à boca). Nesse sentido, uma construção transitiva declarativa em português está mais entrincheirada do que uma construção clivada (do tipo "Foi a Maria que fez isso"). Nessa teoria, portanto, a frequência de uso de uma construção influenciaria no seu processamento neural. É importante observar, entretanto, que, assumindo a existência de um procedimento recursivo inato, 
como fazem os gerativistas, a frequência de uma construção não deveria influir em seu processamento. Qualquer experimento sobre a realidade neural da sintaxe, portanto, tem que partir de uma posição teórica sobre esse tipo de questão.

Para os linguistas cognitivos, não é claro se existe um objeto teórico que possa ser classificado como sintaxe. As restrições de ordem das palavras poderiam ser explicadas simplesmente como produto de uma série de comportamentos ensaiados (e entrincheirados). Mesmo que se possa falar em algo parecido com sintaxe, nessa teoria, ela está fora, externa ao falante. Entretanto, a LC não é externalista quando à semântica. Na verdade, a teoria se propõe a explicar o conhecimento humano, e a cognição, numa perspectiva inteiramente internalista.

Apesar do sucesso irrefutável da LC para explicar certos fenômenos linguísticos, essa teoria mantém elementos de uma perspectiva comportamentalista, defendida por filósofos como Quine, para quem, durante a aquisição da língua, "[...] dependemos estritamente da observação de comportamentos observáveis." (QUINE, 1990, p.37). Chomsky (2000a) se contrapõe a esse tipo de argumento, como tem feito historicamente (ver, por exemplo, a crítica contundente de Chomsky (1967) ao livro Verbal Behavior, de B. F. Skinner). De fato, o que se sabe sobre a aquisição da linguagem por crianças normais vai de encontro a essa visão em que os dados observáveis determinariam a linguagem infantil. De maneira geral, as crianças produzem formas lexicais e construções sintáticas ausentes da linguagem adulta. Os exemplos mais comuns são as generalizações morfológicas e os chamados root infinitives. No primeiro caso, documentado em várias línguas, crianças produzem formas finitas de verbos que não existem na língua dos pais (como "fazi", em vez de "fiz", e "sabo", em vez de "sei", em português; e "goed", em vez de "went", em inglês). No segundo, crianças produzem orações sem qualquer marcação morfológica de tempo e concordância, como no exemplo abaixo, do holandês (apud BLOM, 2008, p.18) e do francês (PIERCE, 1992, p.8), respectivamente:

(2) a. Jij de walvis maken.

Você a baleia fazer.

b. Pas rouler en vélo.

Não rolar em bicicleta.

Dados como esses indicam que as crianças não usam uma estratégia do tipo monkey-see-monkey-do; ao invés, as crianças generalizam regras e passam por fases em que sua competência difere daquela do adulto. ${ }^{12}$

12 A linguística cognitiva teria, ainda, de dar conta do que é comumente chamado de "argumento da pobreza do estímulo" (BERWICK et al., 2011; PIATTELLI-PALMARINI, 1980; CHOMSKY, 1957) e da gênesis de línguas 
Mesmo não aceitando esses argumentos e mantendo uma aprendizagem da sintaxe por correlação, um pesquisador precisaria supor (para depois confirmar) uma maneira de instanciar as construções no cérebro. Ou seja, como afirma Pulvermüller (2002), precisa haver um desenvolvimento teórico para que se chegue a respostas que são, em última análise, empíricas. Para ilustrar o problema, tomemos a sentença citada anteriormente "gatos tomam leite". Um indivíduo que ouve (ou lê) essa sentença teria as redes lexicais correspondentes a "gato", "tomar" e "leite" ativadas no seu cérebro, uma após a outra. De que maneira a ocorrência sequencial dessas redes lexicais instancia ou produz, na mente desse sujeito, a construção [NP V NP]? Pulvermüller (2002) aposta em redes neurais detectoras de sequências, que seriam ativadas à medida que cada item lexical é ativado e possibilitariam a identificação de construções por meio de reverberação da atividade neural dessas redes. Apesar dessa descrição esquemática, pode-se concluir que há ainda um longo caminho a ser percorrido para que essas questões possam ser respondidas.

\section{Conclusões}

Chomsky (1980) discute a questão de como a presença de uma representação invisível poderia ser testada por um experimento eletrofisiológico durante o processamento de uma sentença envolvendo um certo tipo de operação sintática (movimento-QU, por exemplo). Sua proposta mantém que, se um certo padrão de atividade elétrica está claramente correlacionado com uma certa estrutura (movimento-QU), então, se esse mesmo padrão ocorre durante o processamento de um outro tipo de sentença (construções-tough, por exemplo), isso seria evidência de que essas últimas construções envolveriam uma operação sintática do mesmo tipo usado nas primeiras, comprovando assim uma representação que foi postulada por considerações teóricas (isto é, a presença de um operador-QU na derivação de uma construção-tough). Entretanto, provar que uma certa operação sintática está realmente relacionada a um "certo padrão de atividade elétrica" tem se provado mais difícil do que Chomsky supunha. Em virtude do fato de que itens lexicais parecem ativar redes neurais espalhadas por várias áreas cerebrais, identificar um certo padrão ou uma área cerebral específica que se correlacione com uma certa operação sintática é bem mais difícil do que se supunha na década de 80. Apesar de alguns estudos proclamarem ter encontrado evidências de tal correlação, as conclusões parecem provir de uma tomada de posição teórica que não é única, nem pode, atualmente, ser comprovada e, portanto, constituem mais uma interpretação dos fatos do que uma conclusão imparcial e objetiva.

crioulas (BICKERTON, 1981, 1990). Em ambos os casos, parece haver fortes indícios de uma determinação biológica da linguagem, apesar de outras interpretações serem possíveis (PULLUM; SHOLZ, 2002). 
Apesar de a busca de comprovação empírica depender de uma tomada de posição teórica prévia, seria salutar se os pesquisadores mantivessem em mente que o júri ainda está indeciso quanto às questões mais cruciais sobre a linguagem: a realidade interna ou externa da sintaxe; a contribuição do ambiente externo na definição das estruturas linguísticas, a tensão entre predisposições inatas e aprendizagem, entre outros. O trabalho conjunto de linguistas e neurocientistas é crucial para que estratégias de pesquisas sobre essas questões sejam achadas.

\section{Agradecimentos}

Este trabalho é parte do projeto da Universidade de São Paulo "Matemática, Computação, Linguagem e o Cérebro" (MaCLinC) e só foi possível em virtude das discussões com os pesquisadores Antônio Galves, André Frazão Helene, Sidarta Ribeiro, Cláudia Vargas e Nivaldo Vasconcelos, a quem agradeço.

MODESTO, M. Internalism and externalism in linguistics and the neuroscience of language. Alfa, São Paulo, v.58, n.1, p.137-164, 2014.

- ABSTRACT: The main objective of this study is to discuss the possibility of investigating the neurophysiology of the natural languages syntax. In order to develop this kind of analysis, linguists and neuroscientists would have to find a common epistemological basis. However, nowadays, the prevalent view in neuroscience, which assumes associative learning by correlation, does not mesh well with the internalist view of syntax assumed by generative linguists. For that reason, this research discusses the epistemological bases of the most accepted linguistic theories, that perform as a guide for neuroscientists wanting to investigate language processing by the brain. On the other hand, this article also discusses experiments within the neuroscience of language in order to evaluate the kind of problems concerning the intersection of these two areas. The main conclusion is that any researcher who is willing to investigate such problems must have a clear understanding of those epistemological questions before setting up any kind of experiment; and that any conclusion with respect to the neural reality of syntax is, at the present, unwarranted.

- KEYWORDS: Linguistics. Cognition. Neuroscience. Syntax.

\section{REFERÊNCIAS}

BAK, T. H. et al. Selective impairment of verb processing associated with pathological changes in Brodmann areas 44 and 45 in the motor neuron diseasedementia-aphasia syndrome. Brain, Oxford, n.124, p.103-120, 2001.

BEN-SHACHAR, M. et al. The neural reality of syntactic transformations: evidence from functional magnetic resonance imaging. Psychological Science, Thousand Oaks, n.14, p.433-440, 2003. 
BEN-SHACHAR, M.; PALTI, D.; GRODZINSKY, Y. Neural correlates of syntactic movement: converging evidence from two fMRI experiments. NeuroImage, Orlando, n.21, p.1320-1336, 2004.

BERWICK, R. C. et al. Poverty of stimulus revisited. Cognitive Science, Norwood, n.35, p.1207-1242, 2011.

BICKERTON, D. Language and human behavior. Seattle: University ofWashington Press, 1995.

Language and species. Chicago: University of Chicago Press, 1990. Roots of language. Ann Harbor: Karoma, 1981.

BLOCK, N. The computer model of the mind. In: OSHERSON, D. N.; SMITH, E. E. (Org.). An invitation to Cognitive Science. Cambridge: MIT Press, 1990. v.3. p.247-289.

BLOM, E. The acquisition of finitesness. Berlim: Mouton de Gruyter, 2008.

BROWN, C. M.; HAGOORT, P.; TER KEURS, M. Electrophysiological signatures of visual lexical processing: open- and closed-class words. Journal of Cognitive Neuroscience, Cambridge, n.11, p.261-281, 1999.

CAPPA, S. F. et al. Object and action naming in Alzheimer's disease and frontotemporal dementia. Neurology, Minneapolis, n.50, p.351-355, 1998.

CHALMERS, D. Facing up to the problem of consciousness. Journal of Consciousness Studies, Devon, n.2, p.200-219, 1995.

CHOMSKY, N. Language and other cognitive systems: what is special about language? Language Learning and Development, Philadelphia, n.7, p.263-278, 2011.

. On nature and language. Cambridge: Cambridge University Press, 2002.

. New horizons in the study of language and mind. Cambridge: Cambridge University Press, 2000a.

Linguistics and brain science. In: MARANTZ, A. et al. (Org.). Image, language and brain. Cambridge: MIT Press, 2000b. p.13-28.

Rules and representations. New York: Columbia University Press, 1980.

A review of B. F. Skinner's verbal behavior. In: LEON, A. et al. (Org.). Readings in the Psychology of Language. New Jersey: Prentice-Hall, 1967. p.142143.

Syntactic structures. The Hague: Mouton, 1957. 
CHOMSKY, N.; BERWICK, R. The Biolinguistic program: the current state of its evolution and development. In: Di SCIULLO, A. M.; AGUERO, C. (Org.). Biolinguistic investigations. Cambridge: MIT Press. No prelo.

CHURCHLAND, P. M. Matter and consciousness. Cambridge: MIT Press, 1984.

DAMASIO, H. et al. A neural basis for lexical retrieval. Nature, London, n.380, p.499-505, 1996.

DE ARAUJO, D. B. et al. Seeing with the eyes shut: neural basis of enhanced imagery following ayahuasca ingestion. Human Brain Mapping, Hoboken, v.33, n.11, p.2550-2560, nov. 2012.

DEACON, T. The symbolic species: the co-evolution of language and the brain. New York: Norton, 1998

DENNETT, D. Explaining the "Magic" of consciousness. Journal of Cultural and Evolutionary Psychology, Budapest, n.1, p.7-19, 2003.

Consciousness explained. Boston: Little, Brown, 1991.

DEVLIN, J. T. et al. Is there an anatomical basis for category-specificity? Semantic memory studies in PET and fMRI. Neuropsychologia, Oxford, n.40, p.54-75, 2002.

EMBICK, D.; POEPPEL, D. Mapping syntax using imaging: prospects and problems for the study of neurolinguistic computation. In: BROWN, K. (Org.). Encyclopedia of Language and Linguistics. Oxford: Elsevier, 2003. p.484-486.

FODOR, J. The mind doesn't work that way. Cambridge, Mass.: MIT Press, 2000. The language of thought. New York: Crowell, 1975.

FREUD, S. Uma neurose infantil e outros trabalhos (1917-1918). Rio de Janeiro: Imago, 1996. (Coleção Standard Brasileira, 17).

FRIEDERICI,A. D.; PFEIFER, E.; HAHNE, A. Event-related brain potentials during natural speech processing: effects of semantic, morphological and syntactic violations. Cognitive Brain Research, Amsterdam, n.1, p.183-192, 1993.

FUSTER, J. M. Hebb's other postulate at work on words. Behavioral and Brain Sciences, Cambridge, n.22, p.288-289, 1999.

GRABOWSKI, T. J.; DAMASIO, H.; DAMASIO, A. R. Premotor and prefrontal correlates of category-related lexical retrieval. NeuroImage, Orlando, n.7, p.232243, 1998.

GRODZINSKY, Y. The language faculty, broca's region, and the mirror system. Cortex, Varese, n.42, p.464-468, 2006. 
HAUK, O.; PULVERMÜLLER, F. Neurophysiological distinction of action words in the frontal lobe: an ERP study using minimum current estimates. Human Brain Mapping, Hoboken, v.21, p.191-201, 2004.

HAUSER, M. D.; CHOMSKY, N.; FITCH, W. T. The faculty of language: what is it, who has it, and how did it evolve? Science,Washington, n.298, p.1569-1579, 2002.

HEIM, I.; KRATZER, A. Semantics in generative grammar. Oxford: Blackwell Publishers, 1998.

INDEFREY, P. et al. Syntactic processing in left prefrontal cortex is independent of lexical meaning. NeuroImage, Orlando, n. 14, p.546-555, 2001.

JACKENDOFF, R. Foundations of language, brain, meaning, grammar, evolution. New York: Oxford University Press, 2002.

JOHNSON-LAIRD, P. N. The computer and the mind: an introduction to cognitive science. Cambridge: Harvard University Press, 1988.

KIEFER, M.; SPITZER, M. The limits of a distributed account of conceptual knowledge. Trends in Cognitive Sciences, Oxford, n.5, p.469-471, 2001.

LANDAUER,T. K.; DUMAIS, S. T. A solution to Plato's problem: the Latent Semantic Analysis theory of acquisition, induction, and representation of knowledge. Psychological Review, Washington, n.104, p.211-240, 1997.

LÉVI-STRAUSS, C. Structures élémentaires de la parenté. Paris: Presses Universitaires de France, 1949.

MONTAGUE, R. Formal Philosophy. selected papers of Richard Montague. Organizado por e com introdução de Richmond H. Thomason. London: Yale University Press, 1974.

MOORE, C. J.; PRICE, C. J. A functional neuroimaging study of the variables that generate category-specific object processing differences. Brain, Oxford, n.122, p.943-962, 1999.

$\mathrm{MORO}, \mathrm{A}$. The boundaries of babel, the brain and the enigma of impossible languages. Cambridge: MIT Press, 2008.

$\mathrm{MORO}, \mathrm{A}$. et al. Syntax and the brain: disentangling grammar by selective anomalies. NeuroImage, Orlando, n.13, p.110-118, 2001.

MUMMERY, C. J. et al. Functional neuroanatomy of the semantic system: divisible by what? Journal of Cognitive Neuroscience, Cambridge, n.10, p.766-777, 1998.

NEVILLE, H. et al. Syntactically based sentence processing classes: evidence from event-related brain potentials. Journal of Cognitive Neuroscience, Cambridge, n.3, p.151-165, 1991. 
NOBRE, A. C.; McCARTHY, G. Language-related EPRs: scalp distributions and modulation by word type and semantic priming. Journal of Cognitive Neuroscience, Cambridge, n.6, p.233-255, 1994.

NOPPENEY, U.; PRICE, C. J. Retrieval of visual, auditory, and abstract semantics. NeuroImage, Orlando, n.15, p.917-926, 2002.

PENFIELD,W.; RASSMUSSEN,T. The cerebral cortex of man. New York: Macmillan, 1950.

PENFIELD,W.; ROBERTS, L. Speech and brain mechanisms. Princeton: Princeton University Press, 1959.

PIATTELLI-PALMARINI, M. (Org.). Language and Learning: the debate between Jean Piaget and Noam Chomsky. Cambridge: Harvard University Press, 1980.

PIERCE, A. Language Acquisition and Syntactic Theory: a comparative analysis of French and English child grammars. Dordrecht: Kluwer Academic Publishers, 1992.

POEPPEL, D.; EMBICK, D. Defining the relation between linguistics and neuroscience. In: CUTLER, A. (Org.). Twenty-first century psycholinguistics: four cornerstones. New Jersey: Lawrence Erlbaum Associates Publishers, 2005. p.103-118.

PULLUM, Y. K.; SCHOLZ, B. C. Empirical assessment of stimulus poverty arguments. The Linguistic Review, Dordrecht, n.19, p.9-50, 2002.

PULVERMÜLLER, F. The neuroscience of language. Cambridge: Cambridge University Press, 2002.

Brain reflections of words and their meaning. Trends in Cognitive Sciences, Oxford, n.5, p.517-524, 2001.

PULVERMÜLLER, F.; HÄRLE, M.; HUMMEL, F. Neurophysiological distinction of verb categories. Neuroreport, Oxford, n.11, p.2789-2793, 2000.

PULVERMÜLLER, F.; HUMMEL, F.; HÄRLE, M. Walking or talking?: behavioral and neurophysiological correlates of action verb processing. Brain and Language, San Diego, n.78, p.143-168, 2001.

PULVERMÜLLER, F.; LUTZENBERGER, W.; PREISSL, H. Nouns and verbs in the intact brain: evidence from event-related potentials and high-frequency cortical responses. Cerebral Cortex, Cary, n.9, p.498-508, 1999.

PULVERMÜLLER, F.; MOHR, B.; SCHLEICHERT, H. Semantic or lexico-syntactic factors: what determines word-class specific activity in the human brain? Neuroscience Letters, Limerick, n.275, p.81-84, 1999. 
PULVERMÜLLER, F. et al. Brain rhythms of language: nouns versus verbs. European Journal of Neuroscience, Oxford, n.8, p.937-941, 1996.

PUTNAM, H. What is innate and why: comments on the debate. Disponível em: <http://www.federaljack.com/ebooks/Consciousness\%20Books\%20Collection/ Putnam\%20-\%20What\%20Is\%20Innate\%20and\%20Why\%20Comments\%20on\%20 the\%20Debate.pdf>. Acesso em: 11 mar. 2011.

QUINE, W. Pursuit of truth. Cambridge: Harvard University Press, 1990.

SAUSSURE, F. Curso de linguística geral. Tradução de Antônio Chelini et al. São Paulo: Cultrix, 1971.

SHEAR, J. (Org.). Explaining consciousness: the 'hard problem'. Cambridge: MIT Press, 1997.

SHTYROV,Y.; PULVERMÜLLER, F. Memory traces for inflectional affixes as shown by the mismatch negativity. European Journal of Neuroscience, Oxford, n.15, p.1085-1091, 2002.

WARBURTON, E. et al. Noun and verb retrieval by normal subjects: studies with PET. Brain, Oxford, n.119, p.159-179, 1996.

WARRINGTON, E. K.; MCCARTHY, R. A. Category specific access dysphasia. Brain, Oxford, n.106, p.859-878, 1983.

WARRINGTON, E. K.; SHALLICE, T. Category specific semantic impairments. Brain, Oxford, n.107, p.829-854, 1984.

Recebido em agosto de 2012.

Aprovado em fevereiro de 2013. 